The main sources of stray light that may interfere with astronomical observations are:

. lighting of industrial sites, airports and building sites;

road and street lighting;

. advertising signs

. floodlighting of buildings, discos and monuments

. lighting of billboards

lighting of greenhouses

. lighting of sports facilities

. area lighting of sales areas, parking lots, farm yards, railroad yards etc.

The Commission Internationale de l'Eclairage CIE set up 'Guidelines for minimizing sky glow;

A CIE Technical Report'--presently in print. Most recommendations are expressed in values of the Upward Light Output Ratio-installed (ULORinst). The recommendations are based on three principles:

* the requirements for upward light emission are related to the activities in the 'zone' under consideration ('zoning');

* the requirements for upward light emission are most stringent in the 'night'; they may be relaxed in the 'evening' ('curfew');

* the lighting requirements in nearby zones must be taken into account (distance relations).

The future plans for the CIE Technical Committee CIE TC 4-21--in close collaboration with IAU Commission 50-include:

preparing Draft ISO-Standards for measuring methods and for limiting values of sky glow preparing a draft manual on the theory and practice of road lighting for astronomers and for highway authorities.

. collect routine sky brightness measurements from various observatories contribute to the education of astronomy and the role of lighting there-in.

\title{
7. U.S PERSPECTIVE FOR INTERFERENCE TO RADIO ASTRONOMY
}

\section{T. E. Gergely (Division of Astronomical Sciences, National Science Foundation)}

During the last decade a number of new radio telescopes were built, and several are currently in various construction, design or planning stages. These instruments represent a substantial scientific investment, and radio astronomy continues to contribute many new ideas and results to astronomy. At the same time, the regulatory environment related to the radio spectrum has undergone considerable liberalization in the USA, as well as internationally. In the USA responsibility for spectrum utilization is shared by the National Telecommunication and Information Administration (NTIA), that oversees Government uses, and the Federal Communication Commission (FCC), that looks out for private sector spectrum needs. US spectrum management principles, as stated by the FCC, include the fostering of competition, flexibility of spectrum use, licensing and spectrum fee policies geared towards supporting the value of the spectrum and looking out for both the global market. context and the public interest. Regulatory support of radio astronomy under the last item on this list is certainly not very high among FCC priorities. Similar situation prevails at the International Telecommunication Union (ITU), which regulates the uses of the radio spectrum internationally.

The last decade also has seen an expansion of satellite based radio services. Satellite technologies with substantial spectrum demands include the mobile satellite services, digital audio broadcasting, direct-to-home broadcasting and advanced fixed and fixed satellite services. This last name is a euphemism for satellite systems currently planned for distributing Internet and related material and other high data rate applications. The large expansion of satellite services, simultaneously with the relaxation of regulations, threatens radio astronomy. Considerable spectrum has been reallocated to satellite downlinks, and unavoidably (and sometimes avoidably) these are found next or near enough to radio astronomy bands so that spurious and/or out-of-band emissions spill over, and make astronomical observations difficult or impossible. The increasing spectrum congestion at $\mathrm{cm}$ and $\mathrm{dm}$ wavelengths, the wide bandwidths needed for Internet applications, and the availability of mm-wave technology are beginning to be felt in the $\mathrm{mm}$ and sub-mm regions of the spectrum, which astronomers considered their exclusive domain until recently, regardless of the allocation table.

Solutions to these problems will not be easy. Astronomers will need to educate the general public, as well as the regulatory authorities. In doing so, they must keep in mind that many new 
and satellite based services are extremely popular. The astronomy community will also have to get more involved in the various regulatory fora, and establish good contacts with industry. To do so will require that more human as well as financial resources be dedicated to spectrum management activities.

\section{JAPANESE PERSPECTIVE FOR INTERFERENCE TO RADIO ASTRONOMY}

\section{K. Kawaguchi (NRAO, National Astronomical Observatory)}

Interference to radio astronomical observations becomes serious, especially in relatively low frequency regions. Since general discussions on interference are given in other papers of this conference, the present paper describes some examples of interference detected in recent astronomical observations and future perspective.

I. Spurious in fringe detection between Kashima and VSOP in $1600 \mathrm{MHz}$ region Recently, ISAS (Institute of Space and Aeronautics) launched a spacecraft HALCA with 8-m radio telescope for very long baseline interferometer. The first fringe test was carried out on 1997 May 7 for VSOP (VLBI Space Observatory Program) using the Usuda 64- $\mathrm{m}$ and Kashima 34-m ground radio telescopes and HALCA space telescope. In the test, the Kashima telescope received extended spurious noises at $1642.5 \mathrm{MHz}$, which made it difficult to detect fringes between HALCA and Kashima. Then the observation frequency was shifted to $1670-1686 \mathrm{MHz}$ to avoid spurious in Kashima, and fringes were successfully detected between Kashima and HALCA. The band is protected as radio-astronomical band, but it is noted that such strong spurious are present for interference the astronomical observations.

II. Spurious in Taurus Molecular Cloud-1 observations with Nobeyama 45-m telescope The telescope site Nobeyama was chosen by considering to be free from radio interference. The site is surrounded with mountains and sparsely populated. However, it is found that recent development of various communication systems give large interference to radio observations below $30 \mathrm{GHz}$ region. Especially when we observe quiet, cold dark astronomical source like TMC-1, where spectral line width are narrow as $0.6 \mathrm{~km} \mathrm{sec}{ }^{-1}(60 \mathrm{kHz}$ at $30 \mathrm{GHz})$, it becomes very difficult to discriminate real lines from artificial spurious emission, even if a position switching method is employed for astronomical objects.

III. 95-GHz cloud profiling radar CRL (Communication Research Laboratory, Japan) and NASDA (National Space Development Agency of Japan) have project of a cloud profiling radar system from satellite for investigation of global warming which can be emotive issues. The radar system will use $3 \mathrm{~mm}$ radiation by considering scattering effect and established technology levels.

The frequency is also very important for radio astronomical observations. The following interference will occur,

(1) Main lobe-main lobe coupling

In the case of the Nobeyama 45-m telescope, the received power from the radar is estimated to be about $1 \mathrm{~W}$ by considering the sizes of radar beam and telescope. Since the destruction power for SIS (Superconductor-Insulator-Superconductor) junction is estimated about $10 \mathrm{~mW}$, the radar power is two order of magnitude larger than the destruction power.

(2) Main lobe (telescope)-side lobe (radar) coupling

This is the case that a telescope sees a radar side lobe. Usually an SIS mixer receiver is working with a local power of $10^{-9} \mathrm{~W}$, and higher local power gives a change in bias point and results in increase of noise temperature.

(3) Side lobe-side lobe coupling

This is the case that a telescope receives a power less than receiver saturation level, but the radar radiation is observed as a spurious line at the exact radar frequency. The telescope receives more power than harmful interference level $(\mathrm{Pr}=-203 \mathrm{dBW}$ at $90 \mathrm{GHz}$ region), for a shorter distance than $1080 \mathrm{~km}$. If the satellite radar system wants to use the $95 \mathrm{GHz}$ band, the band must be allocated to active sensing from satellite. From astronomical points of view, we are worry about such thing that many active services use the $95 \mathrm{GHz}$ region after the allocation. The $95 \mathrm{GHz}$ region is also not allocated for radioastronomical bands, but the effects (1) and (2) from radar make it difficult to observe in radio astronomical bands of $3 \mathrm{~mm}$ region. We must make efforts to reallocate the $95 \mathrm{GHz}$ band for astronomical bands, because of protection of the best observing bands. Alternative way is to reserve the millimeter-wave telescope sites as protection zones. 